\title{
ON MONOCHROMATIC SUBGRAPHS OF EDGE-COLORED COMPLETE GRAPHS
}

\author{
Eric Andrews ${ }^{1}$, Futaba Fujie ${ }^{2}$ \\ Kyle Kolasinski ${ }^{1}$, Chira Lumduanhom ${ }^{1}$ \\ AND \\ ADAM YUSKO ${ }^{1}$ \\ 1 Department of Mathematics \\ Western Michigan University \\ Kalamazoo, MI 49008 USA \\ ${ }^{2}$ Graduate School of Mathematics \\ Nagoya University \\ Nagoya, Japan 464-8602 \\ e-mail: eric.s.andrews@wmich.edu \\ futaba@math.nagoya-u.ac.jp \\ kyle.c.kolasinski@wmich.edu \\ chira.lumduanhom@wmich.edu \\ adam.m.yusko@wmich.edu
}

\begin{abstract}
In a red-blue coloring of a nonempty graph, every edge is colored red or blue. If the resulting edge-colored graph contains a nonempty subgraph $G$ without isolated vertices every edge of which is colored the same, then $G$ is said to be monochromatic.

For two nonempty graphs $G$ and $H$ without isolated vertices, the monochromatic Ramsey number $\operatorname{mr}(G, H)$ of $G$ and $H$ is the minimum integer $n$ such that every red-blue coloring of $K_{n}$ results in a monochromatic $G$ or a monochromatic $H$. Thus, the standard Ramsey number of $G$ and $H$ is bounded below by $\operatorname{mr}(G, H)$. The monochromatic Ramsey numbers of graphs belonging to some common classes of graphs are studied.

We also investigate another concept closely related to the standard Ramsey numbers and monochromatic Ramsey numbers of graphs. For a fixed integer $n \geq 3$, consider a nonempty subgraph $G$ of order at most $n$ containing no isolated vertices. Then $G$ is a common monochromatic subgraph of $K_{n}$ if every red-blue coloring of $K_{n}$ results in a monochromatic copy of
\end{abstract}


$G$. Furthermore, $G$ is a maximal common monochromatic subgraph of $K_{n}$ if $G$ is a common monochromatic subgraph of $K_{n}$ that is not a proper subgraph of any common monochromatic subgraph of $K_{n}$. Let $\mathcal{S}(n)$ and $\mathcal{S}^{*}(n)$ be the sets of common monochromatic subgraphs and maximal common monochromatic subgraphs of $K_{n}$, respectively. Thus, $G \in \mathcal{S}(n)$ if and only if $R(G, G)=\operatorname{mr}(G, G) \leq n$. We determine the sets $\mathcal{S}(n)$ and $\mathcal{S}^{*}(n)$ for $3 \leq n \leq 8$.

Keywords: Ramsey number, monochromatic Ramsey number, common monochromatic subgraph, maximal common monochromatic subgraph.

2010 Mathematics Subject Classification: 05C15, 05C35, 05C55.

\section{REFERENCES}

[1] G. Chartrand, L. Lesniak and P. Zhang, Graphs and Digraphs (Chapman and Hall/CRC, Boca Raton, FL., 2010).

[2] S.P. Radziszowski, Small Ramsey numbers, Electron. J. Combin. (2011) DS1.

Received 12 March 2012

Revised 26 September 2012

Accepted 2 October 2012 